\title{
Endophthalmitis after Intraocular Lens Exchange: The Role of Wound Leak
}

\author{
Flynn HW Jr ${ }^{1 *}$, Read SP ${ }^{1}$, Young RC ${ }^{1}$ and Rachitskaya A $^{2}$ \\ ${ }^{1}$ Department of Ophthalmology, Bascom Palmer Eye Institute, University of Miami Miller School of Medicine, Miami, Florida, USA \\ ${ }^{2}$ Department of Ophthalmology, Cole Eye Institute, Cleveland, Ohio, USA
}

${ }^{*}$ Corresponding author: Flynn HW Jr, Department of Ophthalmology, Bascom Palmer Eye Institute, University of Miami Miller School of Medicine, Miami, Florida, USA, Tel:+ 305 326-6118; E-mail: HFlynn@med.miami.edu

Received date: January 27, 2016; Accepted date: April 5, 2016; Published date: April 8, 2016

Citation: Flynn HW Jr, Read SP, Young RC, Rachitskaya A (2016) Endophthalmitis After Intraocular Lens Exchange: The Role of Wound Leak. J Eye Cataract Surg 2:10. doi: 10.21767/2471-8300.100010

Copyright: (c) 2016, Flynn HW Jr, et al. This is an open-access article distributed under the terms of the Creative Commons Attribution License, which permits unrestricted use, distribution, and reproduction in any medium, provided the original author and source are credited.

\section{Abstract}

Acute-onset endophthalmitis after intraocular lens (IOL) exchange is a rare clinical entity. The following series describes two patients who presented with endophthalmitis and light perception vision following IOL exchange and were treated with pars plana vitrectomy and intravitreal antibiotics. Wound leak from the temporal clear corneal incision was noted in both patients. Immediate intravitreal antibiotics and pars plana vitrectomy led to a favorable outcome in one of the two patients.

Keywords: Intraocular lens exchange; Endophthalmitis; Acute-onset postoperative endophthalmitis; Pars plana vitrectomy

\section{Introduction}

Endophthalmitis is a serious and potentially devastating condition that may occur after any intraocular surgery. The incidence of acute-onset postoperative endophthalmitis after cataract surgery ranges from $0.03 \%$ to $0.15 \%$ [1-3]. One study described a rise in the incidence of post-cataract endophthalmitis, and has attributed this increase to wound construction in sutureless clear corneal incision techniques [3]. Several reports have indicated a higher incidence of endophthalmitis after secondary intraocular lens (IOL) and IOL exchange procedures as compared to all other intraocular surgery, including cataract surgery [1]. A case series of endophthalmitis after secondary $\mathrm{IOL}$ procedures at our institution proposed that re-entry through a prior incision and postoperative wound leak were associated with higher rates of postoperative infection [4].

The following case series reports two patients with acuteonset endophthalmitis following IOL exchange in which wound leak was noted.

\section{Report of Cases}

\section{Case 1}

An 86 year old female underwent IOL exchange because of a persistent pupillary membrane refractory to anterior YAG laser capsulotomy of the right eye. The posterior chamber IOL was exchanged via a $7 \mathrm{~mm}$ temporal limbal incision near the site of the prior clear corneal incision. An anterior vitrectomy was performed following the IOLThe patient presented on postoperative day five with pain and decline in vision to light perception. A diagnosis of acute-onset postoperative endophthalmitis was made on clinical examination (Figure 1a). B-scan echography showed diffuse moderate vitreous opacities (Figure 1b).

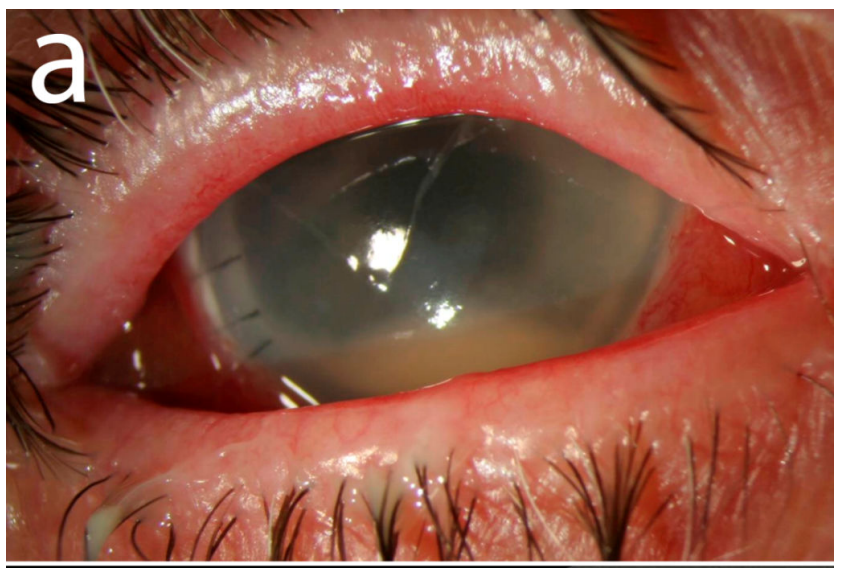

Figure 1a: 86 year old female with acute-onset endophthalmitis after IOL exchange. Slit lamp examination demonstrated a fibrinous anterior chamber reaction with hypopyon. Six interrupted 10-0 nylon sutures were present through the temporal clear corneal incision. 


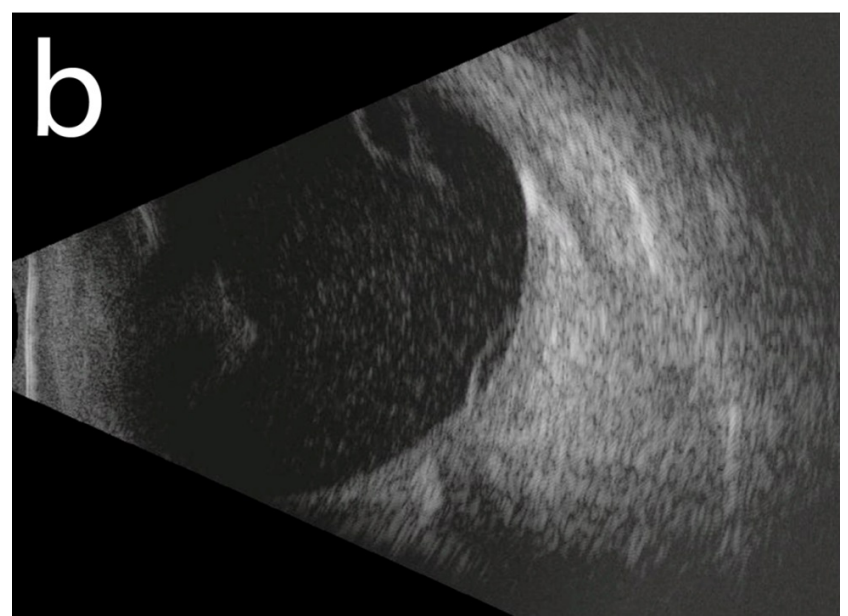

Figure 1b: Contact B-scan ultrasonography demonstrating dense vitreous opacities, membrane formation, and premacular bursa formation.

The patient was treated with pars plana vitrectomy. Vitreous cultures were obtained and intravitreal injections of vancomycin $1 \mathrm{mg} / 0.1 \mathrm{~mL}$, ceftazidime $2.25 \mathrm{mg} / 0.1 \mathrm{~mL}$, and dexamethasone $0.4 \mathrm{mg} / 0.1 \mathrm{~mL}$ were given. Intraoperatively, a wound leak was noted at the temporal clear corneal incision, and the leak was closed with one additional 10-0 nylon suture. Streptococcus viridians were cultured and the patient received an additional intravitreal injection of vancomycin $0.1 \mathrm{mg} / \mathrm{mL}$ on postoperative day one. Initially after treatment, the patient's vision remained light perception but declined to no light perception within eight weeks postoperatively.

\section{Case 2}

A 75 year old male had phacoemulsification and IOL exchange of the left eye at an outside institution seven days prior and presented with eye pain and decreased vision. The cataract surgery was performed using a clear corneal approach to phacoemulsification with a lens placed in the capsular bag. Intraoperative IOL exchange was performed as the surgeon noted a scratch on the optic of the initial IOL. The surgery was otherwise uncomplicated, without report of capsular rupture, vitreous loss, wound leakage or dehiscence. The patient presented to an outside ophthalmologist five days postoperatively, and was diagnosed with acute-onset postoperative endophthalmitis and treated with intravitreal injections of vancomycin and ceftazidime. The patient noted continued deterioration of his vision and presented to our institution with light perception vision for further management at seven days postoperatively. Examination at our institution revealed a temporal clear corneal incision with wound leak and anterior chamber inflammation with hypopyon (Figure 2a).

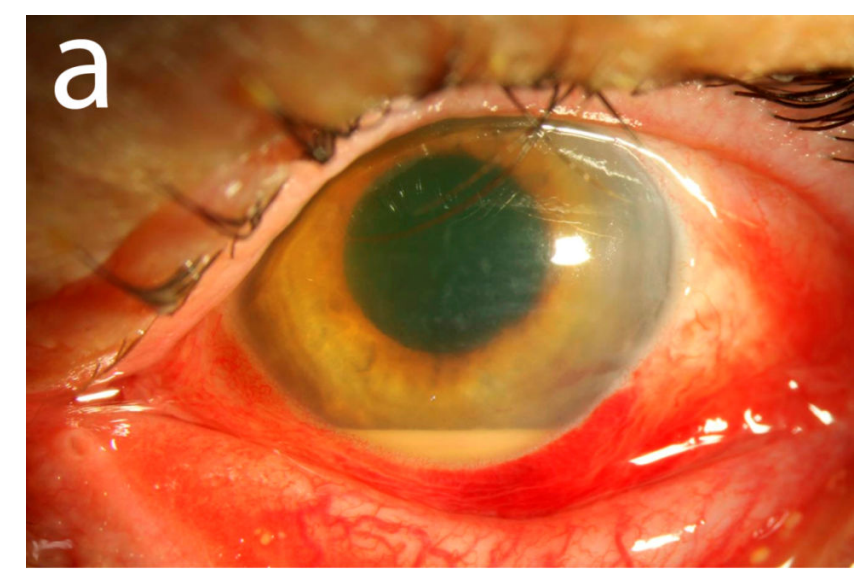

Figure 2a: 75 year old male with acute-onset endophthalmitis after intraoperative IOL exchange. Slit lamp examination revealed corneal edema and an anterior chamber inflammation with hypopyon. A temporal clear corneal incision was noted to be leaking on presentation. No sutures were present in the wound.

A contact B-scan echography demonstrated dense vitreous and sub-hyaloid opacities consistent with endophthalmitis. The patient underwent immediate pars-plana vitrectomy and intravitreal injections of vancomycin $1 \mathrm{mg} / 0.1 \mathrm{~mL}$, ceftazidime $2.25 \mathrm{mg} / 0.1 \mathrm{~mL}$, and dexamethasone $0.4 \mathrm{mg} / 0.1 \mathrm{~mL}$. Intraoperatively, a wound leak was confirmed from the temporal clear corneal incision, and was closed with two interrupted $10-0$ nylon sutures (Figure $2 b$ ).

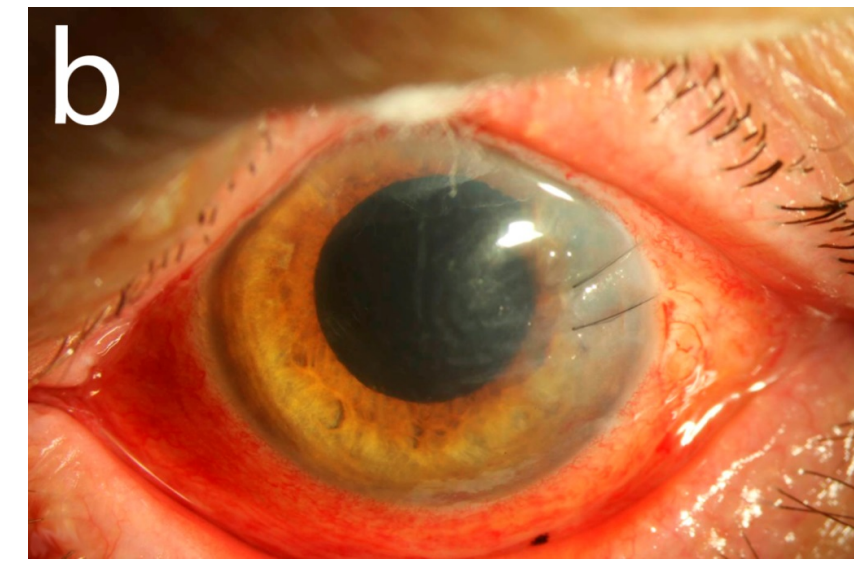

Figure 2b: After pars plana vitrectomy, the follow up exam showed improvement of corneal edema and anterior chamber reaction with resolution of the hypopyon. The temporal clear corneal incision was closed with two interrupted 10-0 nylon sutures. Vision improved to 20/40 ten weeks postoperatively after suture removal.

Vitreous culture isolated methicillin-resistant Staphylococcus epidermidis, which notably was also resistant to levofloxacin and moxifloxacin. After treatment, the patient's vision improved to $20 / 400$ at three days, 20/60 at six weeks and 20/40 at ten weeks 
postoperatively. At final follow up a year and a half later the patient's vision was 20/25 in the left eye.

\section{Discussion}

As patient expectations have continued to rise with cataract surgery, IOL exchange is becoming increasing common. While the role of wound leak has been previously established as a risk factor for endophthalmitis [4], these two cases highlight the importance of wound integrity in preventing this complication. Clear corneal incision cataract surgery has been associated with a higher risk of endophthalmitis compared to scleral or limbal incisions due to poor wound apposition and leak, allowing surface microorganisms entry into the anterior chamber [3,5-7]. In a series of endophthalmitis after secondary IOL procedures, Scott et al. noted wound defects in $50 \%$ of cases [4]. In a randomized control trial evaluating treatments for wound leak, hydrogel sealant showed a significant reduction in wound leakage compared to suture [8].

An experimental study in cadaver eyes showed pressuredependent ingress of India Ink into the anterior chamber through clear corneal wounds [9], and wound gape at low IOP was demonstrated with anterior segment OCT of clear corneal incisions [10]. As the majority of postoperative endophthalmitis is secondary to peri-ocular flora [11], it is likely that poor wound apposition combined with intraocular pressure changes allows the influx of these microorganisms into the anterior chamber. In both patients in the current report, manipulation and enlargement of the temporal clear corneal incision may have led to poor wound apposition and subsequent leak, resulting in endophthalmitis. Several factors play a role in poor wound closure after IOL exchange, including the re-opening of a clear corneal incision, stretching and enlargement of the wound, and difficult closure of the enlarged clear corneal wound. In patients in whom a prior clear corneal incision is re-entered, it is important to evaluate the wound for signs of wound gape or leak. A low threshold for suture placement is necessary if there is uncertainty about wound integrity. Treatment with immediate pars plana vitrectomy and intravitreal antibiotics led to a favourable visual outcome in one of two patients in this series.

\section{Acknowledgement}

Partially supported by NIH Center Core Grant P30EY014801, an Unrestricted Grant from Research to Prevent Blindness, and the Department of Defense (DOD Grant \#W81XWH-09-1-0675).

\section{References}

1. Wykoff CC, Parrott MB, Flynn HW Jr, Shi W, Miller D, et al. (2010) Nosocomial acute-onset postoperative endophthalmitis at a university teaching hospital (2002-2009). Am J Ophthalmol 150: 392-398.

2. Packer M, Chang DF, Dewey SH, Little BC, Mamalis N, et al. (2011) Prevention, diagnosis, and management of acute postoperative bacterial endophthalmitis. J Cataract Refract Surg 37: 1699-1714.

3. Mehran T, Behrens A, Newcomb RL, Nobe MY, Saedi G, et al. (2005) Acute endophthalmitis following cataract surgery: a systematic review of the literature. Arch Ophthalmol 123: 613-620.

4. Scott IU, Flynn HW, Feuer W (1995) Endophthalmitis after secondary intraocular lens implantation: a case- control study. Ophthalmology 102: 1925-1931.

5. Lundstrom M, Wejde G, Stenevi U, Thorburn W, Montan P (2007) Endophthalmitis after cataract surgery: a nationwide prospective study evaluating incidence in relation to incision type and location. Ophthalmology 114: 866-870.

6. Nagaki Y, Hayasaka S, Kadoi C, Matsumoto M, Yanagisawa S, et al. (2003) Bacterial endophthalmitis after small-incision cataract surgery. effect of incision placement and intraocular lens type. J Cataract Refract Surg 29: 20-26.

7. Cooper BA, Holekamp NM, Bohigian G, Thompson PA (2003) Casecontrol study of endophthalmitis after cataract surgery comparing scleral tunnel and clear corneal wounds. Am J Ophthalmol 136: 300-305.

8. Masket S, Hovanesian JA, Levenson J, Tyson F, Flynn HW Jr, et al. (2014) Hydrogel sealant versus sutures to prevent fluid egress after cataract surgery. J Cataract Refract Surg 40: 2057-2066.

9. Taban M, Sarayba MA, Ignacio TS, Behrens A, McDonnell PJ (2005) Ingress of India ink into the anterior chamber through sutureless clear corneal cataract wounds. Arch Ophthalmol 123: 643-648.

10. McDonnel PJ, Taban M, Sarayba M, Rao B, Zhang J, et al. (2003) Dynamic morphology of clear corneal cataract incisions. Ophthalmology 110: 2342-2348.

11. Schimel AM, Miller D, Flynn HW Jr (2013) Endophthalmitis isolates and antibiotic susceptibilities: a 10-year review of culture-proven cases. Am J Ophthalmol 156: 50-52. 